\title{
Research on the Creative Techniques of Animation Scenes Shuai Yuan ${ }^{1}$ \\ ${ }^{1}$ City College of WUST, Wuhan, China 430083 \\ a304695974@qq.com
}

\begin{abstract}
Keywords: Animation scene; Layer concept; Permeability; Separation line; Simple sense
\end{abstract}
\begin{abstract}
The animation scene design is an important link in the early and late stage of animation, which directly influences the quality and style of animation. Animation scene late in the majority with special effects, the early stage of the design is more important, animation scene creation techniques have appeared in the scene design, the concept of layers, dividing line, animation scene composition principle, the colour of the animation scene principle, principle of animation scene permeability are animated scenes creation such as the use of the necessary means to ignore the basic creation techniques, it is hard to the scene of the early creation ascending to a height of more perfect. In the paper mainly expounds the animation scene creation technique, the layer concept study, the relationship between the space motion and texture performance aspects detailed analysis, make creative animation scene more the combination of theory with practice, giving animation scene creation has a more intuitive guidance.
\end{abstract}

\section{Research on the Concept of Animation Scene Creation Techniques}

From the point of view of the painting, the animation scene is similar to the background of the illustration in the way of painting, with the background of the theme and the rendering of anger. But for the animation scene, it has a more important role than the illustration background. Most people know that there is a stage for a show, a stage for a performance, and a cartoon? Of course, it also needs the background stage, without the participation of the animation background, how can it be a complete play? The animation scene and the stage design, need the level to increase the effect, highlight the theme, the foil atmosphere is the main function of the animation scene. From early stage design, simple meeting hall plane stage background isn't able to meet the requirements of the kingdoms, translating into a more novel combinations of stage, the so-called three-dimensional stage system, through the principle of three-dimensional construction stage of the hierarchy is more abundant, not only in the stage of practical structures, the use of multi-level way, in the production of stage design, will also join the three dimensional animation system, make the stage level. Stage of the design method as well as the applicable in the animated film, the concept of hierarchy and animation scene of one of the most important creative elements, to service for character animation, animation scene must be talking about "layer".

Generally before the animation scene, deliberately to scene classification for the first layer, middle, and background of the basic three layers, according to the actual situation, of course, made the scene to have more space, fine feeling, can choose in the former, middle and background layer to add excessive layer, so that we can to add more animation scene scene elements, looks more fullness.

In comparison, at the level of the three kinds of animation scene, the first layer is one of the most important, also is the animation scene in front of a layer, the role can not be ignored in the animation scene, is also one of the most frequently used one.

First of all, the distance between the front, middle and background layers is distinguished, so that the picture space presents an open sense of vision and layering. 


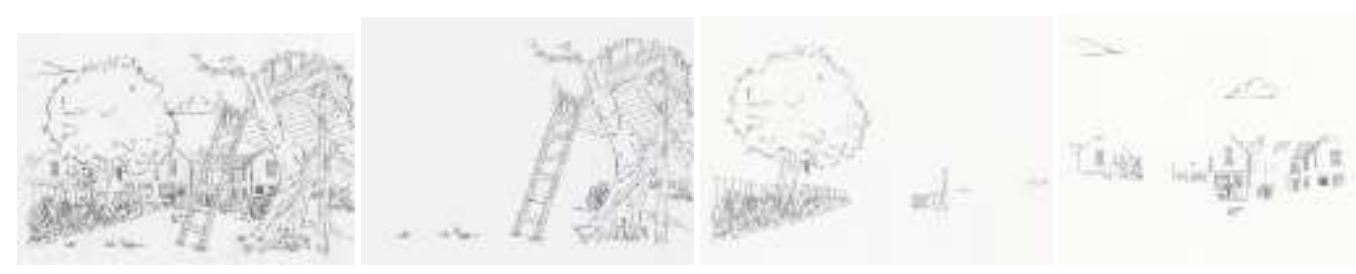

Secondly, the role of the middle and background layers is partially occluded, so that the movement of moving objects can be freely played to make the picture more real. (layer before the picture is compared commonly fine, picture pixel generally high, the requirements of the detail is also quite a lot, edge, multilateral and complex changes, rarely and middle layer before picture, background layer exchange calls in the picture. )

Again, make the picture rich, the layer feeling can make the speed sense and other animation effect.

The edge line of the front layer is also a very important separation line in the animation scene. The selection of separator lines directly influences the composition of the animation scene and the motion trajectory of the moving object. The separation line must be the edge of the previous layer, but the edge line of the front layer is not necessarily the dividing line in the animation scene. The front layer has only one, but the separator line can have many strips. The separator line is one or more lines that overlap between the front layer and the other layers in the animation scene. The main function is to expand the positioning of the moving object and the arrangement of the moving track. Animated film animation scene so much, every animation scene hierarchical processing is required, but particularly large workload, can very quickly in the midst of a layer to separate the first layer, middle, and the content of the background layer, the role of the separation line is particularly important, can be directly by the late software to convert a single animation layer be meet the animation layered scene of animation, so can greatly save time and process, to achieve the same effect. In general, we use red lines to mark the position of the separator line when drawing. The position of the red line must be clearly visible, and the later cutting is basically carried out with its length.

Former layer and the dividing line is not separately using two tools, but to alternating used to be obtained, the two are interrelated, reasonable use of dividing line and the former layer, will be the stage effect of animation scene is more attractive. Before using layer and the separation line, must want to level out the animation scene, former layer, middle, what are the content of the background layer, respectively, the size of the layer in the scene and size is in accordance with the perspective rule, etc.

At the time of drawing animation scene, in many cases will be as a reference to find related entities scene, then elements scenery drawing on the computer or on paper, but in the creation of animation scene, is easy to ignore the feature animation, blindly copy the reference material on their own paper, and as a copier is no effect. At the time of drawing animation scene, not only to consider layer, the problem of the concept of and blend in the traditional painting techniques in creation, including principles of perspective, composition principle, film theory, etc.

\section{The Space and Sports Relationship of Animation Scene Creation Technique}

Animation scene in addition to the layer concept can visually can enlarge a space, outspread space, animation scene mainly come from the perspective of spatial relations, depending on the depth of field, composition principle, etc., of course have a layer of the concept of participation is more perfect. 


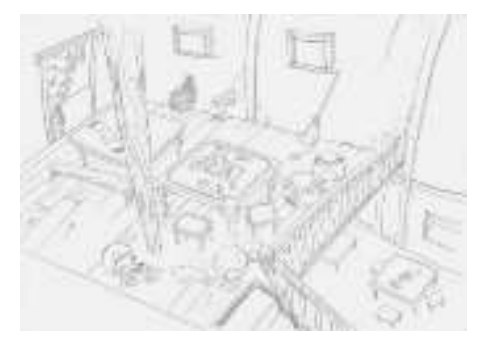

In the construction of spatial relations, "transparency principle" is one of the techniques for the difference of animation scene and general background. The reason why the animation scene is different from the ordinary background is that it requires the characteristic of motion, and the motion is divided into the movement and the scene of the scene to satisfy the two parts of the animation.

The kinematics of the scene: the movement of the scene can be distinguished from the physical features. General animation scenes are divided into static and dynamic scenes by nature. Static scene creation and modelling picture proportion and ordinary TV system aspect ratio is the same as the PAL system and NTSC system, satisfy the general cut scenes and fast lens excessive, use frequency is highest. The appearance of dynamic animation scene is relatively obvious, the ratio of width is not special rules, length-width ratio has the very big difference, shape characteristics similar to horizontal or vertical panoramic photos, long format can simulate the camera movement animations. If on the basis of the effect of mobile and the concept of layers, the scene is not only the movement of the horizontal or vertical plane, you can also create a picture in depth on the shaft of "Z" sensory visual effect, make the dimensional feeling of animation scene better.

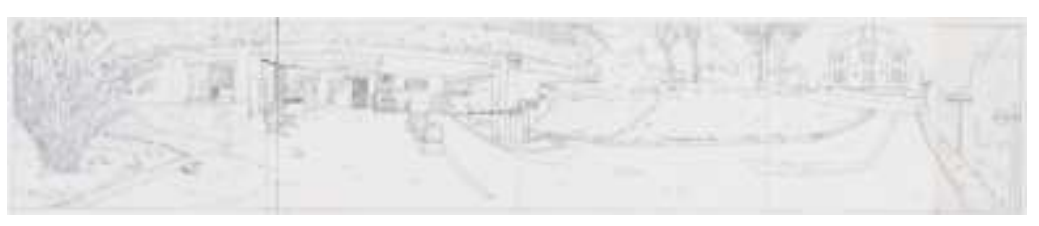

In the scene, the animation is satisfied. The sport in the scene is divided into the following aspects. First, the principle of "permeability". For the animation scene, the principle of "transparency" is the most important point in animation scene, and is the most obvious measure of the animation scene from other backgrounds. "Permeability" principle is at the beginning of the creation of the animation scene line art, on the basis of the combination of animation scene plan, floor plan according to the characters, animals, such as the main body content of trajectory, determine a single lens scene creation of spatial relations, blend composition principles, create a movement, a movement characters through space, have a layer of the concept of scene creation. The characteristics of the principle of "permeability" require that the screen is transparent, and can be divided into various levels of permeability according to the function of the movement track.

\section{Single Permeability Scenario (a Little Perspective)}

The characteristics of single permeability scene in the majority with a little perspective, most of the scene can only provide the role of transverse or longitudinal discrepancy, role becomes big small, the effect of scene in general, there is no clear transparent, transparent point mainly comes from the same camera direction. The animation of the scene and the character is more interesting, and can only be done through the push and pull of the lens. The single permeability scene has no more attention to the concept of separation lines and layers. Generally, there are only two layers, the main layer and the secondary layer, which is the edge line of the front layer or the entire cover. Compared with other permeable scenes, it is monotonous, and the stage configuration provided to the characters is relatively simple. 


\section{Double Permeability Scenario}

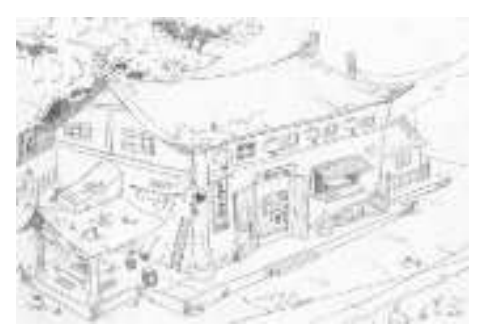

The dual permeability scene is much more complicated than a single permeable scene. It is no longer simply a matter of perspective, but also a consideration of the factors of visual direction and composition. Generally double permeability scenario use two perspectives and three perspectives in the majority, in the process of creation are generally low might look up high and might overlook to render the scene picture, in creating scenes at the same time, general exclusion of angled surface and the Angle is the picture of the central location, such unfavorable to animation performance, would choose gold dividing point to as a general picture of central axis, can not only increase the picture composition aesthetic feeling, can also offer animation role performance of focus.

\section{Multi-permeable Scenes}

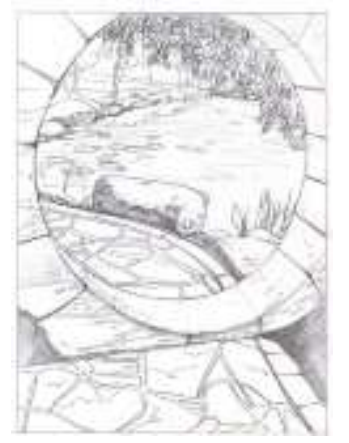

The most important feature of multi-permeable scene is the need to have the participation of the front layer, the camera position needs to be closer to the front layer, and the character's role in the screen is larger, with more central and close-up shots. Such access points can be designed fuzzy roles.

\section{Conclusion}

In numerous animation scene design, experience in the creation of the design method is very effective, and of course there will be a lot of other good design methods and experience, in the later scene design, need more practice to verify and innovation better scenario creation techniques, let everybody in creating animation scene there are more and better ideas, create a more brilliant animation scene.

\section{References}

[1] X. X. Cui: Journal of Chifeng University, Vol. 32 (2016) No. 11, p. 146.

[2] Animation scene design Author: lu feng, ge wenbin, lu mei publishing house: China petrochemical publishing company limited publishing time: July 2017.

[3] The thinking and techniques of animation scene design. Author: Chen guo hongfang publishing house: China media university press release date: January 2011.

[4] Animation scene creation techniques. Author: tian Yang publishing house: Beijing joint publishing company publishing time: May 2013.

[5] New century animation professional course: animation scene design. Author: Chen xianhao, published by fudan university press: September, 2014.

[6] Animation scene production training. Author: Yin junping editor: China renmin university press 
release date: August 2011.

[7] Film and television animation scene and special effects production -- 3ds Max/Vue/AfterBurn/FumeFX. Author: liu gang, deng yi, li huifang: people's post and telecommunications press release date: 2013-02-01.

[8] Material animation scene production tutorial. Author: wang lanchuan, xu qinglan publishing house: southwest normal university press time: sep. 2017.

[9] Digital animation scene design. Author: wang ping, liu qingke, publishing house: China university of science and technology press release date: August 2015.

[10]Li jing. A brief discussion on the analysis and suggestion of the current art training market [J]. 